\title{
Desenvolvimento Perceptomotor e Escrita em crianças do Ensino Fundamental
}

\author{
Adriana Cristina Boulhoça Suehiro \\ Universidade Federal do Recôncavo da Bahia - Cruz das Almas - BA \\ Acácia Aparecida Angeli dos Santos \\ Universidade São Francisco - Itatiba - SP \\ Fabián Javier Marín Rueda \\ Universidade São Francisco - Itatiba - SP
}

\section{Resumo}

O presente estudo verificou quanto o desempenho no B-SPG se relaciona com o desempenho na escrita, buscando por validade convergente entre os instrumentos empregados, bem como eventuais diferenças entre os participantes em relação à idade e ano escolar. Participaram 199 crianças de 7 a 10 anos $(M=8,46, D p=1,15)$, do segundo ao quinto ano do Ensino Fundamental, do interior de São Paulo. Os participantes foram submetidos, coletivamente, ao Bender-Sistema de Pontuação Gradual (B-SPG) e à Escala de Avaliação da Escrita (EAVE). Os resultados confirmaram a relação existente entre o desenvolvimento perceptomotor e a escrita $(r=0,56 ; p<0,001)$, conferindo-lhes uma evidência de validade visto que uma baixa pontuação no B-SPG refletiu um bom desempenho na EAVE e vice-versa. Ficou demonstrado que o nível escolar e a idade são variáveis importantes na avaliação do desenvolvimento perceptomotor e da aprendizagem da escrita. No entanto, novas pesquisas são necessárias a fim de sanar as limitações aqui encontradas.

Palavras-chave: Aprendizagem; escrita; psicometria.

\section{Perceptual motor development and Writing in children of elementary school}

\begin{abstract}
The present study verified the performance as the B-SPG relates to performance in writing, searching for convergent validity between the instruments used and any differences between participants with respect to age and school year. Participated 199 children 7-10 years $(M=8.46$, $S D=1.15)$ from the second to fifth year of elementary school, in the countryside of the state of São Paulo. The participants underwent collectively to-Bender Gradual Scoring System (B-SPG) and the Writing Assessment Scale (EAVE). The results confirmed the relationship between the perceptual motor development and writing $(r=0.56 ; p<0.001)$, giving them validity evidence because of a low score on the B-SPG reflected a good performance in EAVE and vice versa. Evidence shows that the educational level and age are important variables in assessing the perceptual motor development and learning of writing. However, further research is need in order to remedy the limitations found here.
\end{abstract}

Keywords: Learning; writing; psychometrics.

\section{Desarrollo Perceptomotor y Escrita en niños de lo Enseñanza Fundamental}

\section{Resumen}

El presente estudio verificó cuanto el desempeño en el B-SPG se relaciona con el desempeño en la escrita, buscando por validad convergente entre los instrumentos empleados, así como eventuales diferencias entre los participantes y relación a la edad y año escolar. Participaron 199 niños de 7 a 10 años $(M=8,46, D p=1,15)$, del segundo al quinto año de la Enseñanza Fundamental, del interior de São Paulo. Se sometieron los participantes, colectivamente, al Bender-Sistema de Puntuación Gradual (B-SPG) y a la Escala de Evaluación de la Escrita (EAVE). Los resultados confirmaron la relación existente entre el desarrollo perceptomotor y la escrita $(r=0,56 ; p<0,001)$, confiriéndoles una evidencia de validad una vez que una baja puntuación en el B-SPG reflexionó un buen desempeño en la EAVE y vice-versa. Quedó demostrado que el nivel escolar y la edad son variables importantes en la evaluación del desarrollo perceptomotor y del aprendizaje de la escrita. Sin embargo, nuevas investigaciones son necesarias con la finalidad de cubrir las limitaciones aquí encontradas.

Palabras clave: aprendizaje; escrita; psicometría. 


\section{Introdução}

A aprendizagem da escrita demanda a ação conjunta de diversas habilidades, tais como, discriminação auditiva; decodificação dos sons; discriminação visual; organização e orientação dos elementos no espaço; sequência dos movimentos finos e temporal; controle do próprio corpo; noção de lateralidade, entre outros (Drouet, 2003). Dada a complexidade dessa habilidade e sua multideterminação, falhas na sua aquisição podem ser provenientes de condições neurobiológicas, fatores hereditários e condições ambientais desfavoráveis, dentre outros fatores, sendo que as deficiências que mais tendem a causar dificuldades escolares são aquelas que afetam a percepção visual, o processamento da linguagem, as habilidades finas e a capacidade para focalizar a atenção (Drouet, 2003; Santos, \& Jorge, 2007; Smith, \& Strick, 2001).

Em razão disso, o desenvolvimento perceptomotor é considerado importante para a lectoescrita (Snowling, 2004; Suehiro, \& Santos, 2005; entre outros). Sua investigação possibilita não apenas identificar os déficits que poderão acarretar problemas durante o processo de aquisição da leitura e da escrita, mas, sobretudo, favorecer o estabelecimento de metas e estratégias que potencializem suas chances de sucesso. Cunha (1990), por exemplo, verificou que as crianças com alto desenvolvimento psicomotor, por ela avaliadas, estavam mais aptas para a compreensão do sistema de representação necessário para a escrita.

Uma das explicações para a estreita relação entre a escrita e o desenvolvimento visomotor está no fato de que a representação da escrita, assim como a habilidade perceptomotora ou visomotora, demanda alguns princípios da gestalt que regem a forma de o sujeito perceber os estímulos visuais, quais sejam, fechamento, proximidade e continuidade, que são formas primitivas da experiência, biologicamente determinados por um padrão sensório motor de ação e que apresentam um caráter evolutivo. Ao lado disso, há que se destacar que ambas as habilidades demandam uma integração complexa, uma vez que o simples fato de perceber um estímulo não garante ao sujeito a capacidade de copiá-lo. Para que essa representação seja possível, a criança deve transferir sua percepção à atividade motora (Silva, \& Nunes, 2007).

Destarte, a percepção visomotora, fruto do desenvolvimento da criança ou resultante de treinamento e aprimoramento, se consolida com base em quatro etapas, quais sejam, visão do estímulo; compreensão do que se vê (percepção); tradução do que é percebido numa ação ou expressão motora; e coordenação da ação motora. Sendo assim, uma maturação perceptomotora adequada pode ser considerada como uma habilidade facilitadora do processo de aprendizagem e, portanto, um componente importante para a evolução das habilidades acadêmicas, cujo baixo desempenho tende a se relacionar a dificuldades ao longo do processo de aprendizagem (Bender, 1955; Koppitz, 1989; Noronha, Rueda, \& Santos, 2013; Pinto,\& Noronha, 2010; Silva, 2008; Silva,\& Nunes, 2007; Sisto, Santos,\& Noronha, 2010; Suehiro, 2008).
Um dos instrumentos utilizados para a avaliação do desenvolvimento perceptomotor é o Teste Gestáltico Visomotor de Bender. As relações entre leitura, escrita e desenvolvimento perceptomotor, no que concerne à aplicabilidade e validade do Bender como um instrumento preditivo dessas habilidades ainda não estão bem consolidadas na literatura. Sobretudo quando se consideram os resultados provindos de pesquisas realizadas com base no sistema de correção de Koppitz (Bandeira, \& Hutz, 1994; Koppitz, 1975, Mckay, \& Neale, 1985; Nielsen, 1969; Nielson, \& Sapp, 1991; para citar alguns). De maneira geral, os estudos realizados com base no Bender - Sistema de Pontuação Gradual (B-SPG), tanto com relação à leitura e à escrita, quanto à dificuldade de aprendizagem dessas habilidades, têm demonstrado a sensibilidade do sistema em diferenciar os indivíduos nas avaliações realizadas (Carvalho,\& Noronha, 2009; Santos, Suehiro,\& Vendemiatto, 2009; Suehiro, \& Santos, 2005; Suehiro, \& Santos, 2009).

Suehiro e Santos (2005), por exemplo, buscaram por evidência de validade de critério para o B-SPG, ao comparar grupos divididos por dificuldades de aprendizagem (acentuada/média/leve/sem indícios). Participaram do estudo 287 crianças, de ambos os sexos,com idades entre 7 e 10 anos $(M=8,6, D P=0,76)$, provenientes de escolas públicas e particulares. Os instrumentos utilizados pelas autoras foram um questionário de identificação dos sujeitos, a Escala de Avaliação de Dificuldades na Aprendizagem da Escrita (ADA$P E)$ e as nove figuras do B-SPG. Os resultados permitiram a identificação de evidências de validade para o B-SPG, visto que diferenciaram os grupos quanto aos níveis de dificuldade de aprendizagem. Assim, crianças com dificuldades leve, média e acentuada apresentaram mais erros de distorção de forma do que as sem indícios de problemas com a aprendizagem. Além disso, com base no índice de correlação obtido, as autoras concluíram que crianças com problemas na aprendizagem da escrita tendem a apresentar dificuldades perceptomotoras.

No ano seguinte, as autoras realizaram um estudo com a finalidade de averiguar outras evidências de validade para o B-SPG. Para tanto, analisaram 237 protocolos de crianças do terceiro e quarto anos de escolas públicas e particulares, com média de 8,48 anos de idade $(D P=0,50)$. Constataram um incremento significativo do desempenho em razão do ano frequentado, diferenciando a pontuação de crianças do terceiro e quarto anos, de modo que as crianças do quarto ano apresentaram maiores notas em relação às demais. Assim, Suehiro e Santos (2006) corroboram a associação existente entre a maturidade perceptomotora e a fase de escolarização na qual seus participantes se encontravam.

Diferenças significativas relativas ao ano escolar também foram observadas por Suehiro (2008). Participaram 221 estudantes, de ambos os gêneros, entre 6 e 12 anos $(M=8,53, D P=1,40)$, de primeira à quarta série (atuais segundo ao quinto ano) do ensino fundamental de uma escola pública. As crianças obtiveram uma média de 22,47 erros ( $\underline{\mathrm{DP}}=14,72)$, com uma pontuação mínima de um e máxima de 55 pontos na Escala de Avaliação da Escrita (EAVE), a 
mesma utilizada no presente estudo. A pontuação mais frequente foi 11 , sendo que $50 \%$ dos participantes obtiveram pontuação abaixo de 19 pontos. O teste de Tukey separou as séries em três grupos, mostrando que a terceira e a quarta série não se diferenciaram entre si, mas se diferenciaram das demais. Ao lado disso, evidenciou um decréscimo no número de erros e, portanto, melhores desempenhos com o avançar da escolaridade.

Também no que concerne às idades dos participantes, a autora verificou diferenças estatisticamente significativas. No entanto, não se evidenciou, conforme o esperado, um decréscimo no número de erros obtidos pelas crianças mais velhas, tendo em vista que os participantes com 10 anos ou mais obtiveram uma média menor quando comparados aos de $8(M=24,25)$ e 7 anos $(M=34,53)$, respectivamente, porém, superior $(M=14,92)$ aos de 9 anos $(M=14,15)$ e, portanto, um número maior de erros no instrumento utilizado.

No que se refere aos resultados obtidos no B-SPG, Suehiro (2008) também encontrou diferenças significativas nas médias obtidas por idade e por ano escolar.As crianças obtiveram uma média de 9,49 erros $(D P=3,78)$, com uma pontuação mínima de 1 e máxima de 19 pontos no Bender. As pontuações mais frequentes foram 7 e 9 , sendo que $50 \%$ dos participantes obtiveram pontuação abaixo de 9 pontos. Os resultados obtidos e a distribuição das frequências evidenciaram uma tendência de pontuações entre 5 e 13 pontos. O teste de Tukey, semelhantemente à compreensão em leitura e à aprendizagem da escrita, separou as séries em três grupos, indicando que a terceira e a quarta série se diferenciaram da segunda e da primeira, porém estas não se diferenciaram entre si. Ao lado disso, evidenciou um decréscimo no número de erros e, portanto, uma evolução do grafismo com o avançar da escolaridade.

Diferentemente do observado em relação à compreensão em leitura e à aprendizagem da escrita, houve um decréscimo significativo no número de erros apresentados pelas crianças mais velhas, uma vez que os participantes de 10 anos ou mais obtiveram uma média menor $(M=7,19)$ quando comparados aos de $9(M=7,94), 8(M=10,70)$ e 7 anos $(M=12,02)$, respectivamente. Quando analisada a correlação entre os instrumentos, o B-SPG apresentou coeficientes moderados e significativos em relação a outros instrumentos utilizados na pesquisa, reforçando sua relação com as habilidades de leitura e escrita.

Embora não tenham empregado a Escala de Avaliação da Escrita, Carvalho, Noronha, Pinto e Luca (2012) também verificaram a relação existente entre desenvolvimento perceptomotor e a escrita, identificando, a exemplo dos estudos descritos anteriormente, diferenças significativas em sua amostra no que concerne ao ano escolar e à idade. Carvalho e cols. (2012) investigaram evidências de validade para o Bender - Sistema de Pontuação Gradual, pela relação com o Teste de Reconhecimento de Palavras e buscaram verificar se o Bender é sensível para captar diferenças de média por idade e escolaridade. Participaram do estudo297 crianças, com idade entre7 e 10 anos $(\underline{M}=9,04, \underline{D P}=0,88)$, que cursavam do segundo ao quinto anos do ensino funda- mental de escolas particulares $(27,3 \%)$ e públicas $(72,7 \%)$ do interior do estado de São Paulo. O B-SPG apresentou correlação negativa com o Reconhecimento de Palavras $(\underline{r}=-$

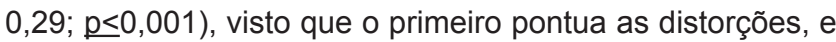
o segundo, os acertos. $O$ índice obtido apresentou-se com magnitude baixa, confirmando que os construtos estudados estão relacionados, porém não se pode afirmar que tratem de um único domínio teórico.

A análise correlacional também foi empregada para verificar a relação entre os construtos e as variáveis idade e escolaridade. Os valores foram significativos, embora também com magnitudes baixas. No que se refere ao B-SPG, a correlação com a idade mostrou-se maior que com a escolaridade, embora ambas significativas. Os índices obtidos evidenciam o caráter evolutivo do B-SPG e sua relação com as etapas iniciais acadêmicas. Assim os resultados obtidos colaboraram para a confirmação de que o Teste de Bender pode ser utilizado como um instrumento de avaliação, não somente para verificar a maturidade perceptomotora, mas também como mais um recurso para um amplo processo de avaliação, em especial, a avaliação da aprendizagem de leitura e escrita.

Diante do exposto, é possível observar que são poucas as pesquisas que relacionam a maturidade perceptomotora, quando avaliada pelo B-SPG, e a escrita. Assim, buscando ampliar os estudos com essa finalidade, o objetivo deste estudo foi verificar quanto o desempenho no B-SPG se relaciona com o desempenho na escrita, buscando por validade convergente entre os instrumentos empregados, bem como identificar eventuais diferenças entre os participantes no que se refere às variáveis idade e ano escolar.

\section{Método}

\section{Participantes}

Compuseram a amostra 199 crianças com idade variando de 7 a 10 anos $(M=8,46, D p=1,15)$, e que frequentavam do segundo até o quinto ano do Ensino Fundamental de uma escola particular do interior do estado de São Paulo. De forma mais detalhada, foram $57(28,6 \%)$ de sete anos, 44 $(22,1)$ de oito, $48(24,1 \%)$ de nove anos e, por fim, 50 crianças tinham 10 anos, o que representou $25,1 \%$ da amostra. Destaca-se que todas as crianças estavam cursando a série escolar correspondente à sua idade, ou seja, não havia repetentes nas séries. Em relação ao sexo, 106 (53,3\%) eram meninos.

\section{Instrumentos}

Teste Gestáltico Visomotor de Bender - Sistema de Pontuação Gradual (B-SPG) (Sisto, Noronha,\& Santos, 2005)

O instrumento é composto por nove figuras $(A, 1,2,3$, $4,5,6,7$ e 8 , sendo que na Figura 7 são analisados os dois 
hexágonos) apresentadas uma a uma para serem copiadas pelo sujeito em uma folha de papel em branco. A instrução dada é de que as crianças façam cópia dos nove modelos em uma única folha de papel, da melhor forma possível, sem a utilização de borracha ou qualquer outro auxílio mecânico. A aplicação pode ser coletiva, por meio de retroprojetor, ou individual, utilizando as lâminas específicas. O B-SPG pode ser aplicado em crianças de seis até os 10 anos de idade.

No B-SPG atribui-se uma pontuação que varia de zero a três pontos, de acordo com a gravidade do erro. Isso possibilita ter um total de 21 pontos, uma vez que as pontuações nas Figuras A, 1, 2, 3, 4, 5, 7a, 7b e 8 podem variar de 0 a 2, enquanto que na Figura 6 a pontuação pode variar de 0 a 3 pontos. Os erros correspondem à presença de desvios em cada uma das figuras do teste. No caso de não existirem desvios relacionados à distorção da forma é atribuído zero ponto.

O teste apresenta estudos de validade e de precisão. Nos estudos de validade destaca-se uma correlação negativa e significativa $(r=-0,58 ; p<0,001)$ entre a idade e a pontuação na prova, confirmando as asserções teóricas. Esse resultado indica que quando aumenta a idade diminui a pontuação obtida no B-SPG, ou seja, as distorções na reprodução das figuras diminuem progressivamente de acordo com o aumento da idade.

No que se refere às diferenças de média entre os grupos de idades e à possibilidade de discriminação entre eles, na ANOVA o resultado mostrou a idade como a fonte de maior variância, sendo a única que apresentou diferenças estatisticamente significativas. Em relação ao sexo, a variável não se mostrou fonte de variância suficiente para produzir diferenças que não sejam atribuídas ao acaso. Quanto à precisão, as análises realizadas por meio do alfa de Cronbach, do método das metades de Spearman-Brown e pelo modelo Rasch indicaram índices satisfatórios. Ainda foi estudada a precisão entre avaliadores, evidenciando uma concordância superior a $80 \%$ em todos os casos.

As normas, assim como os estudos referentes às propriedades psicométricas anteriormente descritos, foram realizadas a partir de uma amostra composta por 1052 crianças, provenientes de oito escolas do estado de São Paulo, sendo $51,1 \%$ meninos, e com escolaridade variando da pré-escola até a $4^{a}$ série do Ensino Fundamental (atual quinto ano).

Escala de Avaliação da Escrita (EAVE) (Sisto, 2005)

Trata-se de uma escala com 55 palavras a serem ditadas para crianças de $1^{\mathrm{a}}$ a $4^{\mathrm{a}}$ séries (atuais segundo ao quinto anos). Após ouvir a palavra, o aluno deve reproduzi-la, sendo que para cada palavra escrita de forma errada é atribuído um ponto. Dessa forma, o escore máximo possível é de 55 pontos. A escolha das palavras ditadas foi feita com base em um estudo desenvolvido com 3365 escolares de ambos os sexos, provenientes de escolas públicas e particulares do interior do estado de São Paulo. Em relação às evidências de validade, foi realizado um estudo que permitiu identificar quais as palavras que apresentaram uma maior discriminação entre as diferentes séries. Ainda foi obtido um coeficiente de correlação de 0,89 com a Escala de Avaliação de Dificul- dades na Aprendizagem da Escrita (ADAPE), o que permitiu concluir por evidência de validade convergente para a EAVE.

\section{Procedimentos}

Inicialmente, o projeto foi submetido e aprovado por um Comitê de Ética em Pesquisa, sendo posteriormente agendada uma reunião na escola para explicar aos pais ou responsáveis pelas crianças os objetivos da pesquisa. Com a autorização e assinatura do Termo de Consentimento Livre e Esclarecido, procedeu-se à coleta de dados. Os procedimentos adotados para tal seguiram rigorosamente as orientações contidas no material de ambos os instrumentos. A coleta do material foi realizada de forma coletiva, e a ordem de aplicação foi a mesma em todas as salas, sendo inicialmente aplicado o B-SPG e em sequência a EAVE.

No caso do B-SPG as crianças foram solicitadas a copiar, da melhor maneira possível, as figuras desenhadas em transparência que lhes foram apresentadas por meio de um retroprojetor. Por sua vez, na EAVE foi solicitado que as crianças prestassem atenção, pois as palavras não seriam repetidas, assim como foram informadas de que nas palavras que começassem com letra maiúscula, seria falado 'letra maiúscula' e posteriormente seria ditada a palavra. Feitos esses esclarecimentos, cada palavra foi ditada pela professora, com intervalo de cinco segundos entre cada uma delas. O tempo aproximado de aplicação em cada sala foi de 50 minutos.

\section{Análise de dados}

$\mathrm{Na}$ análise dos resultados são apresentadas as estatísticas descritivas de ambos os instrumentos, seguidas de uma comparação de média realizada pela análise de variância. Nesse sentido, no caso do B-SPG foi realizada uma comparação em função da idade e na EAVE a comparação foi em função da série escolar. Essa opção se deu levando em consideração a variável que cada um dos testes considera mais importante para sua avaliação, ou seja, a idade no caso do Bender e a série escolar segundo a EAVE. Também calculou-se a correlação entre a pontuação dos instrumentos, considerando a amostra total e as idades de forma separada e, por fim, os grupos extremos formados em função da pontuação no B-SPG foram comparados no desempenho na EAVE.

\section{Resultados}

Considerando-se os objetivos do presente trabalho, os resultados serão apresentados seguindo-se a ordem em que foram estabelecidos. A Figura 1 mostra que, no caso do Bender, as pontuações variaram entre dois e 19, ou seja, nenhuma criança teve a menor ou a maior pontuação possível (zero e 21 respectivamente). 
Figura 1. Frequência das pontuações no B-SPG e na EAVE.

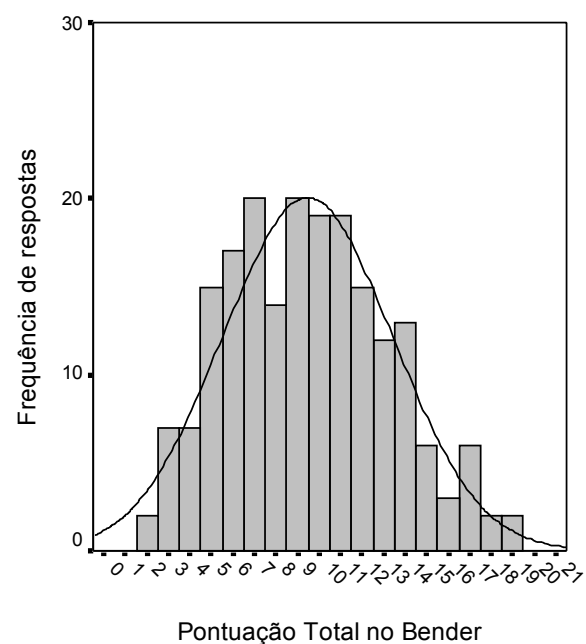

Por sua vez, no caso da EAVE variaram de zero a 55 , evidenciando que houve crianças que não cometeram nenhum erro (2\%), assim como também houve crianças que não acertaram nenhuma das palavras (2\%). Pela Figura 1 também pode ser observado que no B-SPG houve uma distribuição mais próxima do normal do que na EAVE. No que se refere às pontuações, a média no B-SPG foi 9,51 $(d p=3,77)$ e na EAVE 22,28 pontos $(d p=14,88)$. A moda no Bender foi sete pontos, e na EAVE 11.

Dando continuidade, na análise dos dados foram correlacionados os resultados do B-SPG com os da EAVE, considerando a amostra total e as idades de forma separada. Os resultados da análise podem ser observados na Tabela 1.

Observa-se que todas as correlações foram positivas e estatisticamente significativas, com magnitudes variando de fracas a moderadas. Realizou-se, ainda, uma comparação de grupos extremos. Para isso, as crianças foram separadas em função de terem obtido as maiores e as menores pontuações no B-SPG, e posteriormente esses dois grupos foram comparados por meio da prova $\underline{t}$ de student em função do resultado obtido na EAVE. Os resultados mostraram diferenças

Tabela 1. Coeficientes de correlação $(r)$ e níveis de significância $(p)$ entre o B-SPG e a EAVE, por idade e para a amostra total.

\begin{tabular}{|c|c|c|c|c|}
\hline & & \multicolumn{3}{|c|}{ B-SPG } \\
\hline & & $N$ & $r$ & $p$ \\
\hline \multirow{5}{*}{ EAVE } & 7 anos & 57 & 0,27 & 0,044 \\
\hline & 8 anos & 44 & 0,39 & 0,008 \\
\hline & 9 anos & 48 & 0,39 & 0,006 \\
\hline & 10 anos & 50 & 0,40 & 0,005 \\
\hline & Total & 199 & 0,56 & $<0,001$ \\
\hline
\end{tabular}

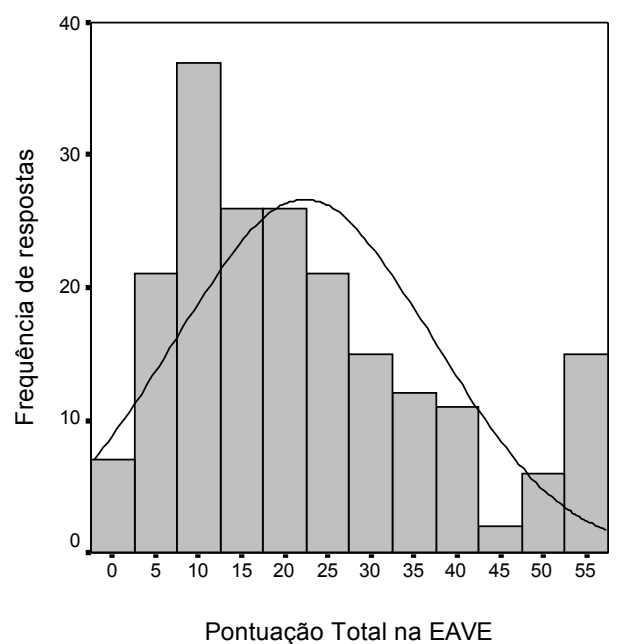

estatisticamente significativas $(t=-9,51, p<0,001)$, sendo que as crianças com as menores pontuações no B-SPG, ou seja, as que tiveram um melhor desempenho no teste tiveram também o melhor desempenho na EAVE, pois a pontuação média (erros cometidos) foi menor do que o grupo com mais pontos no B-SPG (11,96 e 31,96 pontos, respectivamente).

Por fim, foi realizada uma comparação de média no B-SPG em função da idade das crianças. Para isso utilizouse a ANOVA, e os resultados evidenciaram diferenças estatisticamente significativas $[F(198,3)=30,85 ; p<0,001]$. Para verificar a pontuação obtida em quais idades poderia explicar a diferença encontrada, realizou-se a prova de Tukey, e os resultados observam-se na Tabela 2.

Pela prova de Tukey observa-se que houve uma pontuação média maior nas crianças mais novas (7 anos), porém, não houve diferenciação das quatro idades estudadas. As crianças de 7 e 8 anos ficaram congregadas em um agrupamento, enquanto as de 9 e 10 ficaram em outro agrupamento. Ainda, a ANOVA também foi utilizada para comparar a pontuação na EAVE em função do ano escolar

Tabela 2. Prova de Tukey para a pontuação do B-SPG em função da idade das crianças.

\begin{tabular}{cccc}
\hline & \multicolumn{3}{c}{ Subgrupos para alfa $=0,05$} \\
\cline { 3 - 4 } Idade & $N$ & 1 & \\
\hline 10 & 50 & 6,96 & \\
\hline 9 & 48 & 7,94 & 10,70 \\
\hline 8 & 44 & & 12,16 \\
\hline 7 & 57 & & 0,100 \\
\hline$p$ & 199 & 0,409 & \\
\hline
\end{tabular}


Tabela 3. Prova de Tukey para a pontuação da EAVE em função do ano escolar das crianças.

\begin{tabular}{ccccc}
\hline & \multicolumn{4}{c}{ Subgrupos para alfa $=0,05$} \\
\cline { 3 - 5 } Ano escolar & $N$ & 1 & 2 & 3 \\
\hline Quinto & 47 & 11,06 & \\
\hline Quarto & 51 & 15,92 & \\
\hline Terceiro & 54 & & 23,20 & 39,32 \\
\hline Segundo & 47 & & & 1,000 \\
\hline$p$ & 199 & 0,109 & 1,000 &
\end{tabular}

das crianças, sendo verificadas diferenças estatisticamente significativas $[F(198,3)=63,54 ; p<0,001]$. A Tabela 3 apresenta a prova de Tukey, que mostra a pontuação obtida pelas crianças de que ano pode ter explicado essas diferenças.

No caso da EAVE também foi verificado que as crianças dos anos iniciais apresentaram um desempenho mais pobre no instrumento quando comparadas às dos anos finais, o qual melhorou com o passar dos anos de escolarização. Também pode ser observado que, dos quatro anos escolares estudados, apenas três se diferenciaram, sendo que o quarto e o quinto ficaram juntos, enquanto o primeiro e o segundo ano foram separados.

\section{Discussão}

A escrita é uma das formas de linguagem mais requisitadas pela escola. No entanto, para que ela seja possível, muitas são as habilidades necessárias. Nesse sentido, algumas pesquisas têm se dedicado a mostrar que problemas no processamento percepto-visual ou no desenvolvimento perceptomotor podem exacerbar problemas de leitura e escrita (Snowling, 2004; Suehiro, \& Santos, 2005; entre outros). É nesse contexto que o presente estudo se insere. Nele buscou-se verificar quanto o desempenho no B-SPG se relaciona com o desempenho na escrita, bem como identificar eventuais diferenças nas pontuações obtidas pelos participantes em função das variáveis idade e ano escolar.

Considerando que a pontuação máxima possível para o B-SPG é 21 pontos e que o ponto médio do instrumento é 10,5 pontos, bem como que o Bender pontua erros e, portanto, quanto maior é a pontuação, pior é o desempenho, a pontuação média obtida pela amostra aqui pesquisada, a exemplo de outros estudos como o de Suehiro (2008), ficou levemente abaixo da média.Esse resultado demostra que essas crianças apresentam um desenvolvimento perceptomotor considerado adequado. Ao lado disso, observou-se, conforme esperado, um aumento gradual e significativo no desempenho dos participantes em função da idade, uma vez que as crianças de sete anos obtiveram pontuações maiores que as de oito, nove e dez anos, respectivamente. Os resultados obtidos por Carvalho e colaboradores (2012) caminharam nesse mesmo sentido.

Por sua vez, no caso da EAVE também se evidenciou uma pontuação média adequada, tendo em vista que o instrumento pontua igualmente erros, o que indica que as crianças apresentaram uma boa discriminação de fonemas e representação gráfica adequada das palavras ditadas. Esses achados estão em consonância com os observados na pesquisa desenvolvida por Suehiro (2008). Semelhantemente aos estudos de Suehiro (2008), com o EAVE, e de Suehiro e Santos (2006), com o ADAPE (instrumento que deu origem ao EAVE), verificou-se que os alunos dos anos iniciais apresentaram um desempenho mais pobre no instrumento, o qual melhorou significativamente com o passar dos anos.

Os dados relativos à correlação entre os instrumentos empregados revelaram correlações de magnitude fraca, aos sete, oito e nove anos, enquanto que aos dez anos e na amostra total a correlação foi moderada (Dancey, \& Reidy, 2006). Ainda sobre a relação entre os testes na amostra total, se considerado o apresentado por Nunes e Primi (2010), pode-se concluir que foi obtida evidência de validade convergente entre os instrumentos, uma vez que para se considerar como um instrumento que avalie o mesmo construto os coeficientes devem ser de magnitude alta, usualmente acima de 0,51 , além de um dos testes possuir evidências reconhecidas de validade e precisão.

Por fim, a comparação de grupos extremos, em razão das maiores e das menores pontuações no B-SPG, mostrou que as crianças com as menores pontuações no B-SPG, ou seja, as que tiveram um melhor desempenho no teste, também demonstraram o melhor desempenho na EAVE, pois a pontuação média (erros cometidos) foi menor do que o grupo com mais pontos no B-SPG. Suehiro (2008) já havia relatado desempenhos semelhantes.

Embora ainda em número reduzido, diversas pesquisas realizadas anteriormente com base em diferentes instrumentos, entre os quais o ADAPE, instrumento de que derivou o EAVE, já haviam demonstrado a relação que existe entre a aprendizagem da escrita e o desenvolvimento perceptomotor. Os resultados aqui encontrados não apenas confirmam os achados de tais estudos, como afirmam a possibilidade de utilização de outros instrumentos, ampliando, dessa forma, o leque de possibilidades de avaliação dos construtos focalizados. Vale destacar, entretanto, que embora este estudo tenha apresentado uma nova evidência de validade da EAVE, novas pesquisas sobre outras evidências de validade são necessárias para propiciar a ampliação do conhecimento sobre as suas características psicométricas.

Espera-se ainda que a realização de outras pesquisas venha a sanar as limitações deste estudo, utilizando uma amostra maior, bem como crianças de instituições de natureza jurídica diferentes (públicas e particulares), bem como de diferentes regiões e excluir crianças com defasagens. Ao lado disso, tais estudos poderiam incluir outras medidas, tais como de controle da inteligência a fim de verificar a relação 
desses construtos com os aqui estudados. Ao considerar essas sugestões, essas pesquisas poderão, além de contribuir com a ampliação dos conhecimentos na área, favorecer o aprimoramento dos instrumentos e procedimentos técnicos empregados na avaliação psicológica.

\section{Referências}

Bandeira, D. R., \& Hutz, C. S. (1994). A contribuição dos testes DFH, Bender e Raven na predição do rendimento escolar na primeira série. Psicologia: Teoria e Pesquisa, 10(1), 59-72.

Bender, L. (1955). Test Gestáltico Visomotor: usos y aplicaciones clinicas (6a ed.). Buenos Aires: Paidós.

Carvalho, L.,\& Noronha, A. P. P. (2009). Maturidade perceptomotora e compreensão em leitura: um estudo correlacional. Em A. A. A. Santos, E. Boruchovitch, \& K. L. Oliveira (Orgs.), Cloze: um instrumento de diagnóstico e intervenção (pp. 227-247). São Paulo: Casa do Psicólogo.

Carvalho, L., Noronha, A. P. P., Pinto, L. P., \& Luca, L. (2012). Maturidade perceptomotora e reconhecimento de palavras: estudo correlacional entre o Bender - Sistema de Pontuação Gradual e o Teste de Reconhecimento de Palavras. Estudos de Psicologia, 29(3), 371-377.

Cunha, M. F. C. (1990). Desenvolvimento psicomotor e cognitivo: influência na alfabetização de criança de baixa renda. Tese de Doutorado, Universidade de São Paulo, São Paulo-SP.

Dancey, C. P., \& Reidy, J. (2006). Estatística sem matemática para psicologia. Porto Alegre: Artmed.

Drouet, R. C. R. (2003). Distúrbios da aprendizagem (4a ed.). São Paulo: Editora Ática.

Koppitz, E. M. (1975). Bender gestalt test, visual aural digits pan test and reading achievement. Journal of Learning Disabilities, 8(3), 154-157.

Koppitz, E. (1989). O Teste Gestáltico Bender para Crianças. Porto Alegre: Artes Médicas.

McKay, M. F., \& Neale, M. D. (1985). Predicting early school achievement in reading and handwriting using major "error" categories from the Bender-Gestalt test for young children. Perceptual and Motor Skills, 60(2), 647-654.

Nielsen, H. H. (1969). Visuo-perceptive and visuo-motor performance of children with reading disabilities. Scandinavian Journal of Psychology, 10(4), 225-231.

Nielson, S., \& Sapp, G. L. (1991). Bender-gestalt developmental scores: predicting reading and mathematics achievement. Psychological Reports, 69, 39-42.
Noronha, A. P. P., Rueda, F. J. M., \& Santos, A. A. A. (2013). Bender Gestalt Visual-Motor Test Sistema de Pontuação Gradual (B-SPG): A study with different samples. Paidéia, 23, 179-185.

Nunes, C. H. S. S., \& Primi, R. (2010). Aspectos técnicos e conceituais da ficha de avaliação dos testes psicológicos. Em Conselho Federal de Psicologia (Org.), Avaliação psicológica: diretrizes na regulamentação da profissão (pp. 101-127). Brasília: CFP.

Pinto, L. P., \& Noronha, A. P. P. (2010). Maturidade perceptomotora e sua relação com idade e variáveis contextuais: um estudo com o Bender (B-SPG). Encontro: Revista de Psicologia, 13(19), 145-155.

Santos, A. A. A., \& Jorge, L. M. (2007). Teste de Bender com disléxicos: comparação de dois sistemas de pontuação. PsicoUSF, 12(1), 13-21.

Santos, A. A. A., Suehiro, A. C. B., \& Vendemiatto, B. C. (2009). Inteligencia y comprensión en lectura de adolescentes en situación de riesgo social. Paradigma (Maracay), XXX,117-127.

Silva, R. B. F., \& Nunes, M. L. T. (2007). Teste Gestáltico Visomotor Bender: Revendo sua História. Avaliação Psicológica, 6(1), 77-88.

Silva, R. B. F. (2008). Evidência de Validade para o teste Gestáltico Visomotor Bender.Tese de Doutorado, Pontifícia Universidade Católica do Rio Grande do Sul, Rio Grande do Sul-RS.

Sisto, F. F. (2005). Escala de Avaliação da Escrita (EAVE). Relatório Técnico, Universidade São Francisco, Itatiba-SP.

Sisto, F. F., Noronha, A. P. P., \& Santos, A. A. A. (2005). Bender Sistema de Pontuação Gradual B-SPG. São Paulo: Vetor Editora Psicopedagógica Ltda.

Sisto, F. F., Santos, A. A. A., \&Noronha, A. P. P. (2010). Diferential functioning of Bender Visual-motor Gestalt Test itens. Perceptual and Motor Skills, 110, 313-322.

Smith, C., \& Strick, L. (2001). Dificuldades de aprendizagem de a a z: um guia completo para pais e educadores. Porto Alegre: Artes Médicas.

Snowling, M. J. (2004). Dislexia desenvolvimental: uma introdução e visão teórica geral. Em M. Snowling, \& J. Stackhouse (Orgs.), Dislexia, fala e linguagem (pp. 190-213). Porto Alegre: Artmed.

Suehiro, A. C. B. (2008). Processos fonológicos e perceptuais e aprendizagem da leitura e escrita: instrumentos de avaliação. Tese de Doutorado, Universidade São Francisco, Itatiba-SP.

Suehiro, A. C. B., \& Santos, A. A. A. (2005). O Bender e as Dificuldades de Aprendizagem: estudo de validade. Avaliação Psicológica, 4(1), 23-31.

Suehiro, A. C. B., \& Santos, A. A. A. (2006). Bender-Sistema de Pontuação Gradual: avaliando o critério de escolaridade. Interação em Psicologia, 10(2), 217-224. 
Suehiro, A. C. B.\& Santos, A. A. A. (2009). O teste de Cloze e o desenvolvimento perceptomotor no início da escolarização. Em A. A. A., Santos, E. Boruchovitch, \& K. L. Oliveira, (Orgs.), Cloze: um instrumento de diagnóstico e intervenção (pp. 149-164). São Paulo: Casa do Psicólogo.

Recebido em: 14/03/2014

Reformulado em: 20/01/2015

Aprovado em: 15/04/2015

\section{Sobre os autores}

Adriana Cristina Boulhoça Suehiro (dricbs@yahoo.com.br)

Psicóloga. Doutora em Psicologia pelo Programa de Pós-Graduação Stricto Sensu em Psicologia da Universidade São Francisco e docente da Universidade Federal do Recôncavo da Bahia - Santo Antônio de Jesus. Pesquisadora e Líder do Laboratório de Instrumentação e Avaliação Psicológica da Universidade Federal do Recôncavo da Bahia (LABIAP). Bolsista de pós-doutorado na Universidade Estadual de Campinas pelo CNPq.

Acácia Aparecida Angeli dos Santos (acacia.angeli@gmail.com)

Psicóloga. Doutora em Psicologia Escolar e Desenvolvimento Humano pela USP e Docente da Graduação e do Programa de Pós-Graduação Stricto Sensu em Psicologia da Universidade São Francisco/ Itatiba. Bolsista produtividade do CNPq.

Fabián Javier Marín Rueda (arinfabian@gmail.com)

Psicólogo. Doutor em Psicologia pelo Programa de Pós-Graduação Stricto Sensu em Psicologia da Universidade São Francisco e docente do Programa de Pós-Graduação Stricto Sensu em Psicologia da Universidade São Francisco/ Itatiba. Bolsista produtividade do CNPq.

Os autores agradecem o apoio da CAPES. 